Case Report

\title{
Double-layered patella management in total knee arthroplasty for secondary osteoarthritis: A case report
}

\author{
David Eichler, MD, MSc, Pascal-André Vendittoli, MD, MSc, FRCS \\ Department of Orthopaedic Surgery, Université de Montréal, Hôpital Maisonneuve-Rosemont, 5415 boulevard de l'Assomption, Montréal, H1T 2M4, QC, Canada
}

\section{A R T I C L E I N F O}

\section{Keywords:}

Case report

Double-layered patella

Total knee arthroplasty

Patella resurfacing

Multiple epiphyseal dysplasia

Knee osteoarthritis

\begin{abstract}
A B S T R A C T
Double-layered patella (DLP) is an osseous disorder associated with multiple epiphyseal dysplasia (MED). A thorough investigation should be conducted prior to surgery to assess the role of each layer and their vascularization in order to establish the best surgical strategy.

We present the case of a woman in her late 50s with MED, bilateral DLP, and secondary osteoarthritis treated by a left total knee arthroplasty (TKA).

To plan the surgical procedure, bilateral knee magnetic resonance imaging was obtained to study the anatomy of the two bony layers and their relationship to the extensor mechanism. In addition, a computed tomography angiography was performed to study the origins of arterial blood supply to the patella layers to avoid postoperative bone necrosis.

During a standard TKA procedure, after adjusting the thickness of the posterior layer, we fused both layers using outside-in headless compressive screws. Clinical outcome was satisfactory with no anterior knee pain and radiographs showed signs of bone fusion between layers at last follow-up.

No case report was found in the literature in which a full preoperative investigation was made to establish the best surgical management of a DLP during TKA. In such a case, the surgeon should be aware of the distinct roles of both layers (whether in the case of trauma or scheduled surgery) and make sure that the reconstruction plan will provide a favorable outcome.
\end{abstract}

The case

- Double-layered patella (DLP): a challenge for total knee arthroplasty (TKA)?

- What are the options for the two layers: leave both layers untouched, fuse both layers and resurface the posterior layer or fuse both layers with preservation of the articular surface of the posterior layer?

- No scientific published data for best surgical management of DLP in TKA.

- Successful outcome with fusion of anterior and posterior patellar layers with preservation of the articular surface of the posterior layer.

\section{Lessons learnt}

- Thorough preoperative imaging investigation, especially magnetic resonance imaging (MRI), required to avoid vascular and mechanical complications by determining the role of each layer to make the correct decision between fusion, preservation, removal or resurfacing of the patella.

- Quadriceps tendon-patella-patellar ligament relationship: each layer has a purpose.

Abbreviations: ASA, American Society of Anesthesiologists; BMI, Body mass index; CR, Cruciate retaining; CT, Computed tomography; DLP, Double-layered patella;

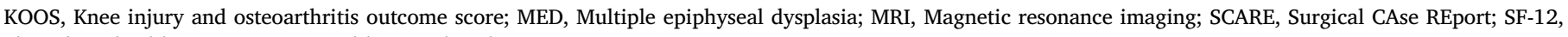
Short form health survey; TKA, Total knee arthroplasty.

* Corresponding author. Tel.: +514 252-3823.

E-mail address: pa.vendittoli@me.com (P.-A. Vendittoli). 


\section{The case}

\section{Introduction}

Double-layered patella (DLP) is found in multiple epiphyseal dysplasia (MED) as well as in non-dysplastic subjects [1]. DLP, first described by Buttner [2], is mostly associated with recessive MED. The frequency of occurrence of DLP/MED is rare: $1 / 20000$ [3]. Patients' symptoms may range from completely asymptomatic to chronic pain due to movements between both layers. Chronic patellar instability is a common symptom [4]. The posterior, intra-articular, layer articulates with the trochlea of the femur while the anterior layer is embedded in the quadriceps tendon. Layers are separated by a coronal septum [5,6]. MED is associated with abnormal contours of the femoral condyles [6] and is a cause of secondary osteoarthritis, especially in hip and knee joints [7].

A thorough review of the literature resulted in 8 case reports describing surgery of a DLP, two involving total knee arthroplasty (TKA) [8-15] (Table 1). No case report was found in the literature in which a full preoperative investigation was made to establish the best surgical management of a DLP. We conducted a thorough investigation to assess the role of each layer and their vascularization in order to establish the best surgical strategy. This work has been reported in line with the updated 2020 Surgical CAse REport (SCARE) criteria [16]. The patient was managed for symptomatic osteoarthritis of the knee in an academic institution specialized in lower limb joint replacement.

\section{Patient information}

\section{Demographic details and presentation}

We report the case of a Caucasian female in her late 50s with MED, referred for bilateral knee osteoarthritis associated with bilateral DLP. The patient presented with knee pain with bilateral genu valgum. Both knees had flexion contractures of $20^{\circ}$ and $130^{\circ}$ of active flexion, with no pathological ligament laxity, a negative lateral patellar apprehension test and no history of patellar dislocation. However, manual mobilization of the anterior layer while the posterior layer was held in place caused discomfort. Body mass index (BMI) was $21 \mathrm{~kg} / \mathrm{m}^{2}(48.6 \mathrm{~kg}, 152 \mathrm{~cm})$. Left knee pain was considered incapacitating, and medical conservative treatment had failed. The preoperative knee injury and osteoarthritis outcome score (KOOS) was 59/168 (Table 2), with zero representing the best score.

\section{Past medical and surgical history}

Medical history included gastroesophageal reflux disease, bilateral L4-L5 neural foraminal stenosis and osteoporosis. Bilateral total hip arthroplasty was performed for osteoarthritis secondary to hip dysplasia. The American Society of Anesthesiologists (ASA) score was 2. No drug allergies were reported.
Table 2

Preoperative and postoperative (15 months) knee injury and osteoarthritis outcome score (KOOS score), with zero representing no knee problems and 168 representing extreme knee problems.

\begin{tabular}{lll}
\hline & preoperative & Follow-up (15 months) \\
\hline KOOS score/168 & 59 & 30 \\
Symptoms/20 & 8 & 10 \\
Stiffness/8 & 3 & 0 \\
Pain/36 & 8 & 2 \\
Function, daily living/68 & 20 & 5 \\
Function, sports and activities/20 & 8 & 12 \\
Quality of life/16 & 12 & 1 \\
\hline
\end{tabular}

\section{Family history}

Parents were second cousins and were not known to have MED. Grandparents and cousins were not known to have MED. One older sibling was totally asymptomatic from MED and one younger sibling was more severely affected by MED than the described patient. Our patient underwent a genetic investigation 30 years ago for fertility purposes, but the results are no longer available.

\section{Social history}

The patient stopped smoking forty years ago, has a sedentary occupation and is capable of walking without stopping on a flat surface and without a walking aid.

\section{Diagnostic assessment and interpretation (Figs. 1-3)}

To select the best treatment for our patient, we had to determine which layer was included in the extensor mechanism of the knee, which layer was functional, and assess the nature of the bond between both layers.

Diagnostic imaging studies consisted of standard radiographs (Fig. 1 a-d), bilateral knee magnetic resonance imaging (MRI) and computed tomography (CT) angiography. These studies demonstrated two distinct anterior and posterior ossified patellar layers on both knees, confirming a diagnosis of bilateral DLP associated with secondary osteoarthritis (Kellgren-Lawrence classification grade 3 [17] and Iwano classification of femoropatellar osteoarthritis stage 2 [18], patellofemoral joint space $\geq 3 \mathrm{~mm}$ ).

Sunrise view demonstrated subluxation of the anterior layer. The lateral view showed a trochlear dysplasia Dejour classification type C [19] (the lateral facet is convex, and the medial facet is hypoplastic, with the crossing sign and double contour sign).

The MRI showed that only the anterior layer was embedded in the extensor mechanism of the knees: quadriceps tendon, lateral and medial patellar retinacula, patellar ligament, suggesting that fusion of both layers was necessary to prevent independent motion of the posterior

Table 1

Case reports involving surgery of double-layered patella.

\begin{tabular}{|c|c|c|c|c|}
\hline Authors & $\begin{array}{l}\text { Year } \\
\text { publication }\end{array}$ & Indication & Surgical management & Patient \\
\hline Przybylski et al. [8] & 2020 & Osteoarthritis & Left TKA: fusion or removal of layers & Adult (47-year-old) \\
\hline Attarzadeh et al. [9] & 2019 & Osteoarthritis & $\begin{array}{l}\text { Bilateral TKA: removal of posterior layer and } \\
\text { resurfacing of anterior layer in one knee }\end{array}$ & Adult (51-year-old male) \\
\hline Jorgensen et al. [10] & 2017 & Fracture & Debridement, tension cerclage, lag screw & Adult (40-year-old male) \\
\hline Nguyen et al. [11] & 2013 & $\begin{array}{l}\text { Anterior knee pain and recurrent painful } \\
\text { catching sensation }\end{array}$ & Fusion with 4 headless screws & Pediatric (15-year-old female) \\
\hline Leng et al. [12] & 2011 & $\begin{array}{l}\text { Intermittent unilateral anterior knee pain and } \\
\text { swelling }\end{array}$ & Single small fragment partially threaded screw & Pediatric (11-year-old female) \\
\hline Goebel et al. [13] & 2009 & $\begin{array}{l}\text { Motion-dependent pain without history of } \\
\text { trauma }\end{array}$ & $\begin{array}{l}\text { Fusion with } 3 \text { double-threaded interference } \\
\text { screws }\end{array}$ & Young adult (17-year-old female) \\
\hline Gardner et al. [14] & 1999 & $\begin{array}{l}\text { Abnormal excursion with a click and a visible } \\
\text { jump in the longitudinal line of movement }\end{array}$ & Compression screw fixation & Pediatric (13-year-old female) \\
\hline Dahners et al. [15] & 1982 & Painful patellofemoral click & Posterior layer removal & Pediatric (12-year-old male) \\
\hline
\end{tabular}

TKA: Total Knee Arthroplasty. 


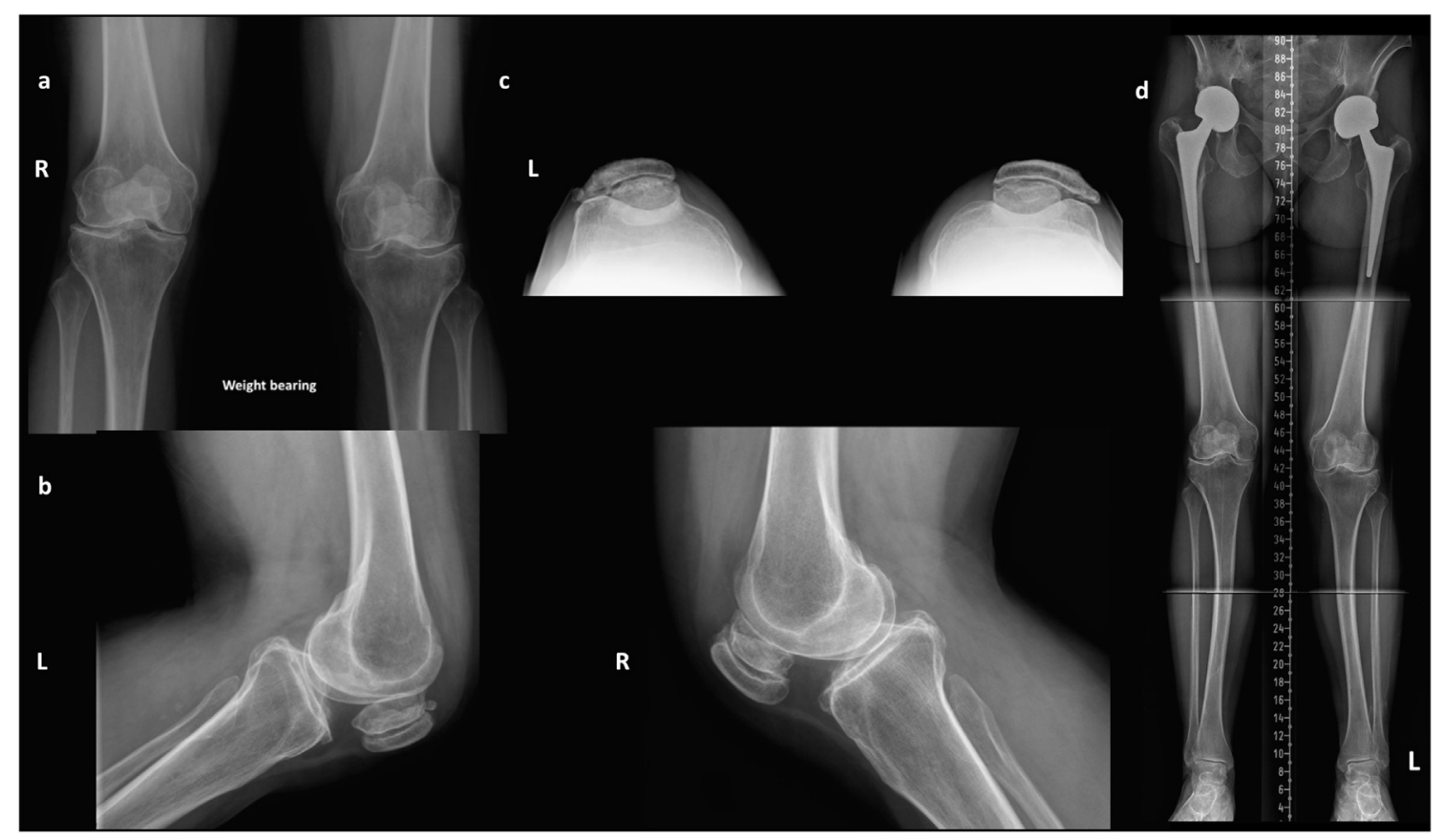

Fig. 1. Standard radiographs showing osteoarthritis and double-layered patella. (a) Antero posterior, (b) Lateral, (c) Patellar view (sunrise), (d) Full length standing radiograph assessing lower limb alignment. Left knee: HKA (hip knee ankle angle) $=186.1^{\circ}$. Mechanical distal femoral angle (mLDFA) $=87.6^{\circ}$. Mechanical proximal tibial angle $(\mathrm{mPTA})=93.6^{\circ}$. Caton-Deschamps index $=0.9(2.4 / 2.6)$ (normal range $\left.0.6-1.2\right)$. Right knee: $\mathrm{HKA}=184.3^{\circ}$, $\mathrm{mLDFA}=93.6^{\circ}$, and $\mathrm{mPTA}=89.8^{\circ}$. Caton-Deschamps index $=0.9(2.4 / 2.6)$.

articular layer. Anterior and posterior cruciate ligaments were intact. Identical findings were described on the right knee MRI (Fig. 2).

A computed tomography angiography (Fig. 3) was performed to map the arterial blood supply for each layer to avoid postoperative bone necrosis and help select a surgical approach minimizing vascular interruption. The right knee has a dominant arterial vascularization for the anterior layer. The medial arterial branch comes from the deep femoral artery and the lateral branch comes from the popliteal artery. The inferior pole of the posterior layer is vascularized by a small branch whose origin is unidentified. Similar observations are made in the left knee with a dominant vascularization for the anterior layer. There are a lateral branch and a medial branch that come from the distal superficial femoral artery.

CT angiography confirmed that we could safely use a midvastus approach without fear of postoperative bone necrosis of one or both layers and that a lateral approach was not mandatory.

\section{Surgical intervention (Fig.4 a to f)}

The patient did not want to have both knees operated on at the same time. General anaesthesia was preferred due to a history of bilateral L4L5 neural foraminal stenosis. The senior surgeon (PAV) and his fellow (DE) performed left TKA by following a day-surgery protocol [22] in an academic institution on October 1, 2019. A midvastus surgical approach was performed to preserve the vascularization of both layers as much as possible. A triathlon cruciate retaining (CR) prosthesis (Stryker Orthopaedics, Mahwah, USA) was cemented (Palacos®, Heraeus Medical) following a kinematic alignment technique, with navigation of the distal femoral and proximal tibial cuts (Stryker navigation system) [23]. No tourniquet was used.

The femoral trochlear groove was shallow. We manually assessed the degree of instability between patellar layers and observed that they moved independently. We measured with a caliper that the patellar

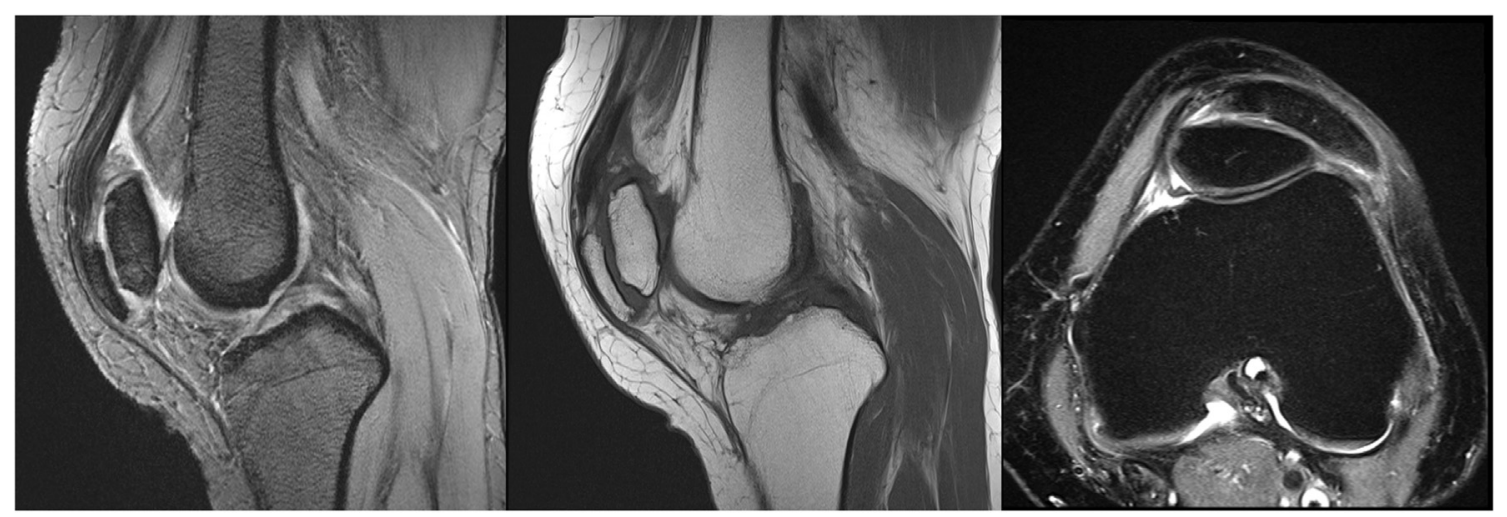

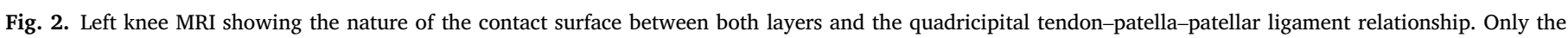

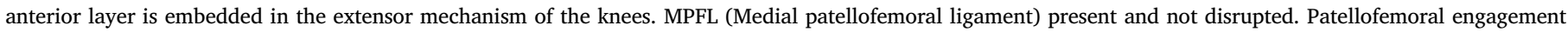
[20]. Patellar ligament length $=4.1 \mathrm{~cm}$ (no length excess according to Neyret et al. [21]). 


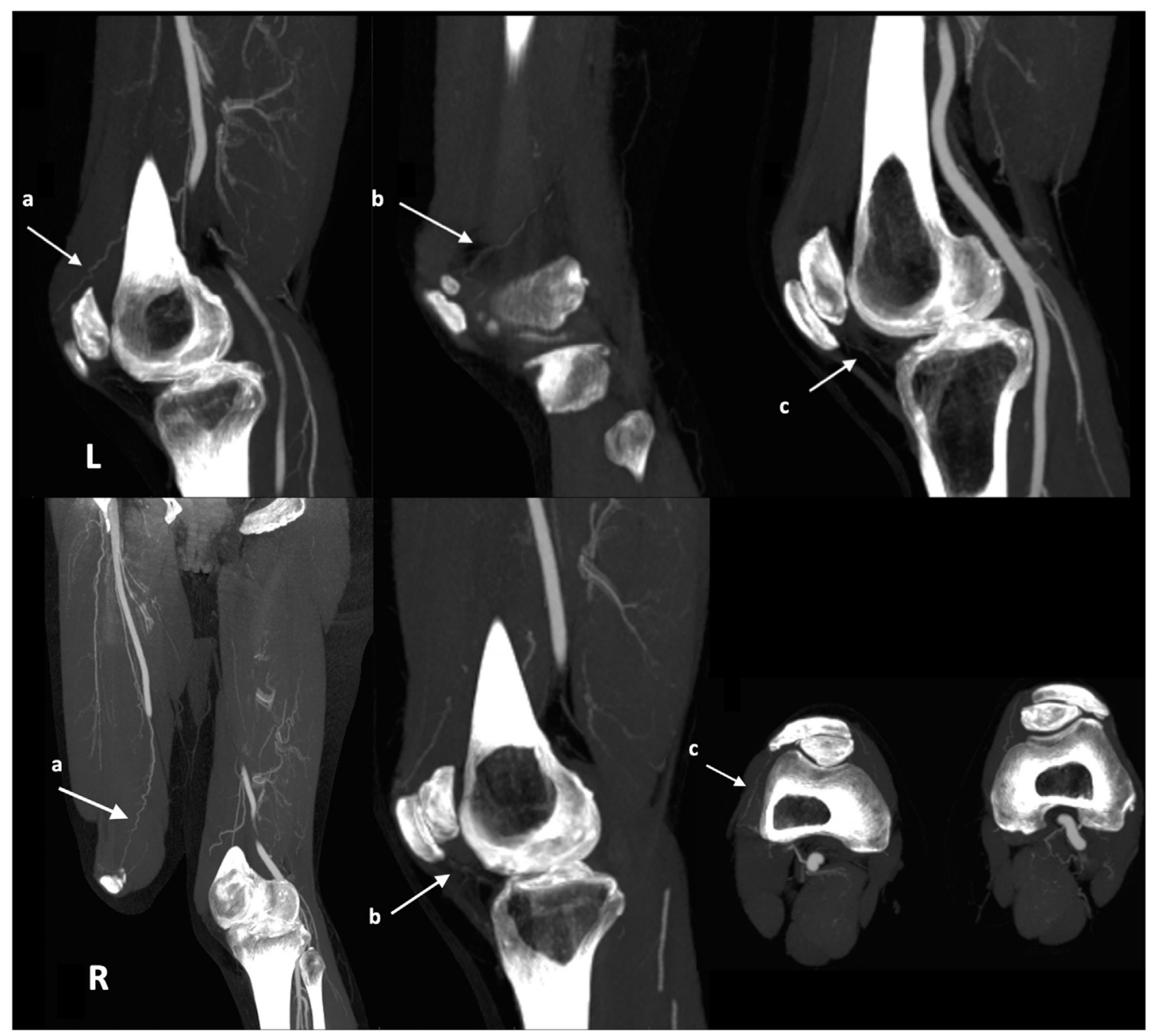

Fig. 3. Computed tomography angiography. Left knee: a: distal superficial femoral artery (supero-medial patellar branch), b: superficial femoral artery (supero-lateral patellar branch), c: small branch towards the posterior layer's inferior pole. Right knee: a: deep femoral artery (medial branch), b: small branch towards the posterior layer's inferior pole, c: popliteal artery (supero-lateral branch).

complex was $27 \mathrm{~mm}$ thick. The two layers were separated from the medial side and the coronal septum was opened like a book. The posterior layer was $20 \mathrm{~mm}$ thick and the anterior $7 \mathrm{~mm}$. Because of its minimal thickness, resurfacing the anterior layer with a polyethylene button would carry too much risk of fracture and would excessively reduce the overall patellar thickness.

A saw was used to remove the synarthrosis-like joint surface on the anterior layer $(-1 \mathrm{~mm})$ and reduce thickness of the posterior layer to $18 \mathrm{~mm}$ for a combined patellar complex of $24 \mathrm{~mm}$. Posterior and anterior layers were aligned and fused with three outside-in $3.5 \mathrm{~mm}$ cannulated compression headless screws: $2 \times 16 \mathrm{~mm}$ and $1 \times 18 \mathrm{~mm}$ (Fixos, Stryker).

\section{Follow-up and outcome}

The patient was discharged the next morning according to our daysurgery protocol $(<24 \mathrm{~h})$. At the 15-month mark, the patient's perception of her knee was that of an artificial joint with no restriction [24]. The knee injury and osteoarthritis outcome score (KOOS) significantly improved during follow up from 59/168 to 30/168 (Table 2). Two KOOS subscales have not improved over follow-up: symptoms and function, sports and activities. The patient occasionally felt some grinding when moving her knee, had moderate difficulty putting on/taking off her socks, and had mild difficulty getting in/out of the bath. Overall, quality of life and activities of daily living significantly improved. In the SF-12, the patient answered that her health was excellent, that she did not feel limited during any activity and had no emotional issues.

Postoperative imaging showed a well-aligned patella with complete fusion of both layers (Figs. 5 and 6). A postoperative $185.5^{\circ}$ valgus hip knee ankle (HKA) angle was measured.

The screws were removed 24 months after the initial surgery only for prophylactic reasons. Since we could not get a good radiograph showing the space between the patella and the femoral component, we were concerned that the articular surface of the patella would wear out and the screws would come into contact with the femoral component.

At the 26-month mark, the patient's perception of her knee and the KOOS score were unchanged.

The patient is now scheduled for the contra-lateral TKA using the same procedure.

\section{Discussion}

DLP represents one of five types of partite patellae [5] that has been previously described in association with MED [15,25] and is caused by a coronal septum that divides the patella into anterior and posterior segments [5]. It should be considered as pathological by radiologists for it is not described as a normal variant of the patella [26]. According to Mäkitie et al. [27], DLP—when present—appears to 


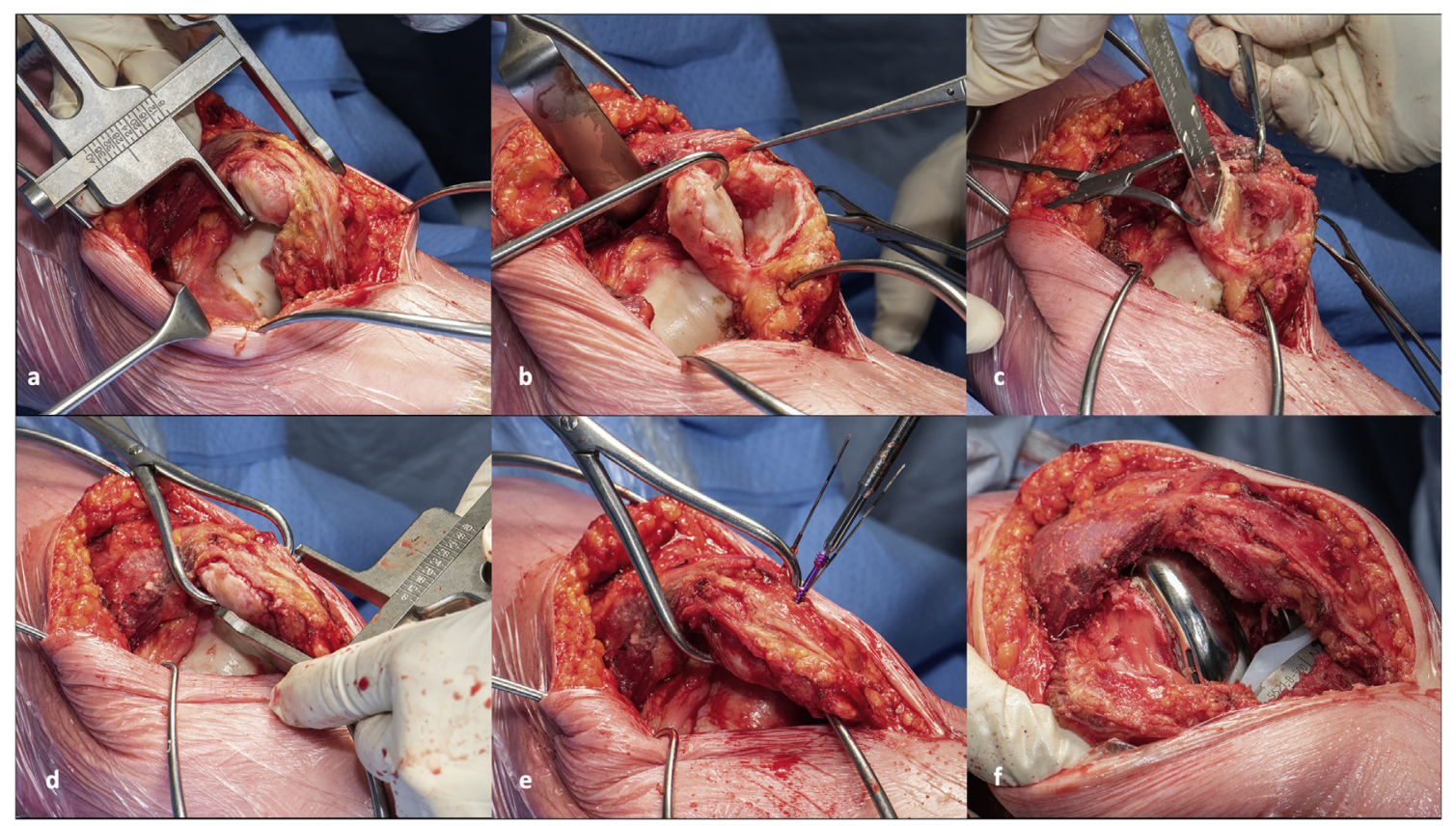

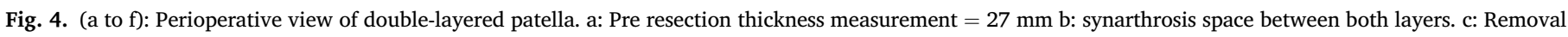

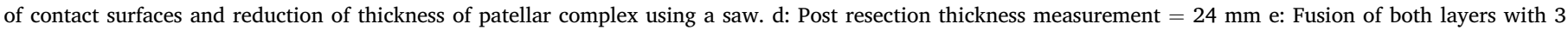
compression headless screws. f: Patellar tracking with final implants in place.

be specific for recessive MED and distinguishes recessive types from autosomal dominant types. In terms of differential diagnosis, it has been proposed that identification of a DLP is highly diagnostic, if not pathognomonic, for MED because DLP has not been described in association with any other skeletal dysplasia or as an isolated abnormality [28].

As confirmed during surgery, the posterior layer was partially mobile and not solidly attached to the quadriceps extensor mechanism [15]. With this finding, a simple resurfacing of the posterior layer was not an option. We measured the exact thickness of each layer. The anterior layer was only $7 \mathrm{~mm}$ thick. Removing the posterior layer and resurfacing the anterior layer with a cemented polyethylene button would present a risk of patellar fracture. From past publications, resurfacing a patella under $12 \mathrm{~mm}$ is not recommended [29].

In the operating room, we were left with three options:

1) Leave both layers untouched.

Patient's patella was very thick $(27 \mathrm{~mm})$, maltracking per operatively and clinically painful. So, this was not our preferred option.

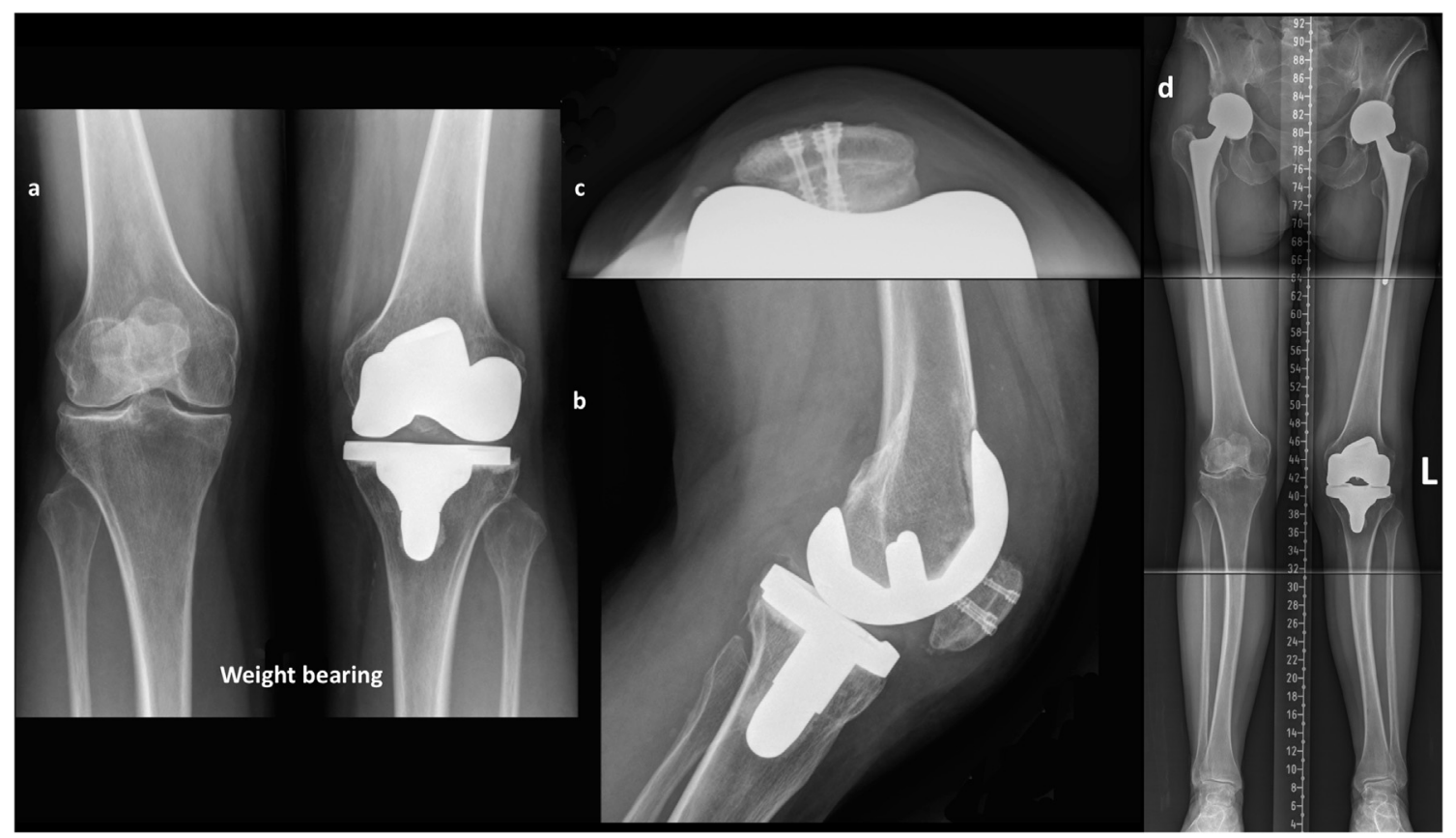

Fig. 5. Postoperative standard radiographs. (a) Antero posterior, (b) Lateral, (c) Patellar view (sunrise), (d) Full length standing radiograph showing appropriate restoration of patient's left lower limb alignment with kinematic alignment. Left lower limb: HKA $=185.5^{\circ}, \mathrm{mLDFA}=88^{\circ}$, and $\mathrm{mPTA}=94.5^{\circ}$. Caton-Deschamps index $=0.9(2.1 / 2.3)$. 


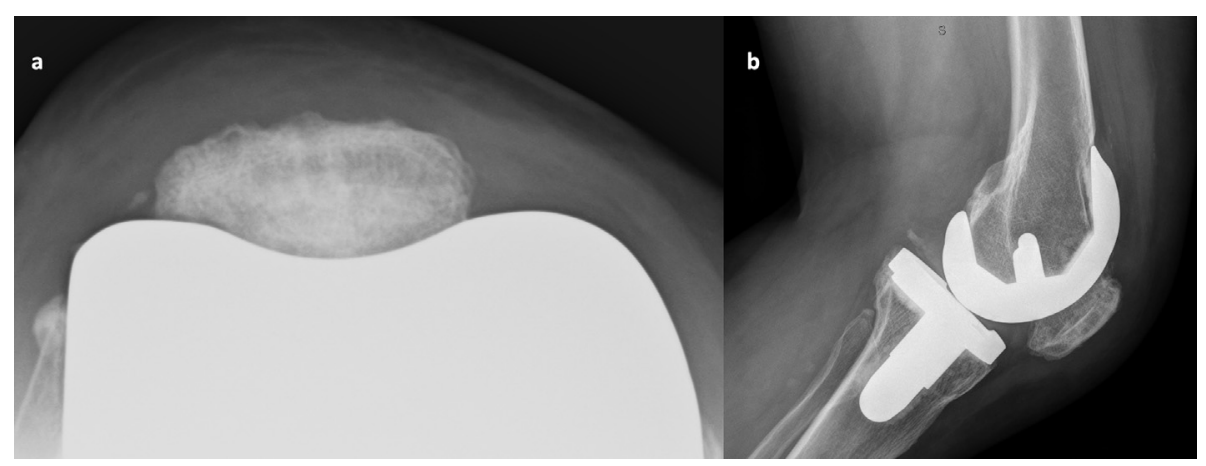

Fig. 6. Standard radiographs at last follow up. (a) Patellar view (sunrise), (b) Lateral. Complete fusion obtained between layers.

2) Fuse both layers and resurface the posterior layer.

Doing so, the thickness of the posterior layer would be less than $10 \mathrm{~mm}$ after removing the patellar button thickness $(9 \mathrm{~mm})$ and some bone to refresh the contact surface between layers. Preserving the vascularization of the thin piece and our ability to fuse it solidly with screws concerned us. Following this decision process, we chose the third option.

3) Fuse both layers and preserve the articular surface of the posterior layer.

The only downside to this option would be in the event of important posterior layer bone resorption/wear, the fixation screw tips might become prominent and cause implant grinding. Secondary patellar resurfacing is still possible should this be deemed necessary on clinical grounds, provided that the screws are removed.

Eight case reports of DLP surgery were found in the literature and only two of them addressed both DLP and TKA (Table 1) [8-15]. Attarzadeh et al. [9] report the case of a 51-year-old male who underwent bilateral TKA. In the left knee, the DLP remained untouched. In the right knee however, the posterior layer was removed, and the anterior layer was resurfaced. Postoperative KOOS score was excellent without any significant difference between both knees. Przybylski et al. [8] do not detail the surgical management of the layers, some bony elements were removed, others were fused. No follow-up was available for the 47-year-old patient.

\section{Conclusion}

A thorough anatomical and functional investigation should be considered before proceeding with surgery on a knee presenting a DLP. The purpose of such an investigation is to determine the role of each layer in order to make the correct decision between fusion, preservation, removal or resurfacing of the patella.

\section{Statement of informed consent}

The described patient was informed that data concerning the case would be submitted for publication and gave her informed consent to the writing of this case report in an anonymized form.

\section{Ethical approval}

The described patient is part of the arthroplasty database of Maisonneuve-Rosemont Hospital (Banque de données d'arthroplastie de l'Hôpital Maisonneuve-Rosemont du CIUSSS de l'Est de l'île de Montréal), which is approved by the ethics committee of Maisonneuve-Rosemont Hospital (CER-CEMTL-2022-2794).

\section{Declaration of competing interest}

PAV reports royalties from Microport inc; grants and personal fees from Stryker; grants and personal fees from Johnson and Johnson; grant from Zimmer outside the submitted work.

$\mathrm{DE}$ reports no relevant competing interest.

\section{References}

[1] García-Mata S, Hidalgo-Ovejero A. Partial double-layered patella in a nondysplasic adolescent. J Pediatr Orthop B 2016;25(6):561-3. https://doi.org/10.1097/ BPB.0000000000000361.

[2] Büttner G. Zur Ätiologie und Pathogenese der perthesschen Krankheit, zugleich ein Fall von Patella tripartita. Arch klin Chir 1925;136:703.

[3] Unger S, Bonafé L, Superti-Furga A. Multiple epiphyseal dysplasia: clinical and radiographic features, differential diagnosis and molecular basis. Best Pract Res Clin Rheumatol 2008;22(1):19-32. https://doi.org/10.1016/j.berh.2007.11.009.

[4] Hinrichs T, Superti-Furga A, Scheiderer WD, Bonafé L, Brenner RE, Mattes T. Recessive multiple epiphyseal dysplasia (rMED) with homozygosity for C653S mutation in the DTDST gene-phenotype, molecular diagnosis and surgical treatment of habitual dislocation of multilayered patella: case report. BMC Musculoskelet Disord 2010;11:110. https://doi.org/10.1186/1471-2474-11-110.

[5] Sheffield EG. Double-layered patella in multiple epiphyseal dysplasia: a valuable clue in the diagnosis. J Pediatr Orthop 1998;18(1):123-8. pmid: 9449113.

[6] Rubenstein JD, Christakis MS. Case 95: fracture of double-layered patella in multiple epiphyseal dysplasia. Radiology 2006;239(3):911-3. https://doi.org/ 10.1148/radiol.2393032020.

[7] Sayilir S, Ekiz T. Early osteoarthritis and double-layered patella in a patient with multiple epiphyseal dysplasia. Arch Rheumatol 2017;32(3):260-3. https://doi.org/ 10.5606/ArchRheumatol.2018.6415.

[8] Przybylski P, Skoczyński M, Tarkowski P, Tarczyńska M, Gawęda K, Drop A. Bilateral double-layered patella in a patient with advanced knee osteoarthritis. Folia Morphol (Warsz) 2021;80(3):735-8. https://doi.org/10.5603/FM.a2020.0089.

[9] Attarzadeh AP, West C, Poulsen KAR. Knæalloplastik hos en patient med bilateral dobbeltlaget patella. Ugeskr Laeger 2019;181(46):V03190175. pmid: 9449113.

[10] Jørgensen A, Brandt E, Ulstrup A. Traumatic separation of osseous segments in a double-layered patella. Int J Surg Case Rep 2017;41:352-5. https://doi.org/ 10.1016/j.ijscr.2017.11.006.

[11] Nguyen MD, Everhart JS, May MM, Flanigan DC. Bilateral double-layered patella: MRI findings and fusion with multiple headless screws: a case report. JBJS Case Connect 2013;3(2):e50. https://doi.org/10.2106/JBJS.CC.L.00314.

[12] Leng HC, Foster P, Templeton PA. Fusion of double-layered patella using a single small fragment partially threaded screw: a case report. J Pediatr Orthop 2011; 31(2):e9-12. https://doi.org/10.1097/BPO.0b013e318207454b.

[13] Goebel S, Steinert AF, Barthel T. Surgical management of a double-layered patella: a case report. Arch Orthop Trauma Surg 2009;129(8):1071-5. https://doi.org/ 10.1007/s00402-008-0701-z.

[14] Gardner J, Woods D, Williamson D. Management of double-layered patellae by compression screw fixation. J Pediatr Orthop B 1999;8(1):39-41. pmid: 10709596.

[15] Dahners LE, Francisco WD, Halleran WJ. Findings at arthrotomy in a case of double layered patellae associated with multiple epiphyseal dysplasia. J Pediatr Orthop 1982;2(1):67-70. https://doi.org/10.1097/01241398-198202010-00010.

[16] Agha RA, Franchi T, Sohrabi C, Mathew G, Kerwan A, SCARE Group. The SCARE 2020 guideline: updating consensus surgical CAse REport (SCARE) guidelines. Int $J$ Surg 2020;84:226-30. https://doi.org/10.1016/j.ijsu.2020.10.034.

[17] Kellgren JH, Lawrence JS. Radiological assessment of osteo-arthrosis. Ann Rheum Dis 1957;16(4):494-502. pmid: 13498604.

[18] Iwano T, Kurosawa H, Tokuyama H, Hoshikawa Y. Roentgenographic and clinical findings of patellofemoral osteoarthrosis. With special reference to its relationship to femorotibial osteoarthrosis and etiologic factors. Clin Orthop Relat Res 1990; 252:190-7. pmid: 2302884.

[19] Dejour H, Walch G, Neyret P, Adeleine P. [Dysplasia of the femoral trochlea]. Rev Chir Orthop Reparatrice Appar Mot 1990;76(1):45-54. pmid: 2140459. 
[20] Dejour D, Ferrua P, Ntagiopoulos PG, et al. The introduction of a new MRI index to evaluate sagittal patellofemoral engagement. Orthop Traumatol Surg Res 2013; 99(8 Suppl):S391-8. https://doi.org/10.1016/j.otsr.2013.10.008.

[21] Neyret P, Robinson AHN, Le Coultre B, Lapra C, Chambat P. Patellar tendon lengththe factor in patellar instability? Knee 2002;9(1):3-6. https://doi.org/10.1016/ s0968-0160(01)00136-3.

[22] Vendittoli PA, Pellei K, Desmeules F, et al. Enhanced recovery short-stay hip and knee joint replacement program improves patients outcomes while reducing hospital costs. Orthop Traumatol Surg Res 2019;105(7):1237-43. https://doi.org/ 10.1016/j.otsr.2019.08.013.

[23] Blakeney WG, Vendittoli PA. Restricted kinematic alignment: the ideal compromise? In: Rivière C, Vendittoli PA, editors. Personalized hip and knee joint replacement. Cham: Springer; 2020. p. 197-206. https://doi.org/10.1007/978-3030-24243-5_17.

[24] Eichler D, Beaulieu Y, Barry J, Massé V, Vendittoli PA. Perception of a natural joint after total knee arthroplasty. J Arthroplasty 2020;35(2):358-63. https://doi.org/ 10.1016/j.arth.2019.09.036.

[25] Hodkinson HM. Double patellae in multiple epiphysial dysplasia. J Bone Joint Surg Br 1962;44-B(3):569-72. https://doi.org/10.1302/0301-620X.44B3.569.
[26] Keats TE. Atlas of normal roentgen variants that may simulate disease. 9th ed. Saunders; 2012.

[27] Mäkitie O, Savarirayan R, Bonafé L, et al. Autosomal recessive multiple epiphyseal dysplasia with homozygosity for C653S in the DTDST gene: double-layer patella as a reliable sign. Am J Med Genet 2003;122A(3):187-92. https://doi.org/10.1002/ ajmg.a.20282.

[28] Goldman AB. Collagen disease, epiphyseal dysplasias, and related conditions. In: Resnick D, editor. Diagnosis of bone and joint disorders. Philadelphia, Pa: Saunders; 2002. p. 4421-9.

[29] Putman S, Boureau F, Girard J, Migaud H, Pasquier G. Patellar complications after total knee arthroplasty. Orthop Traumatol Surg Res 2019;105(1, Supplement): S43-51. https://doi.org/10.1016/j.otsr.2018.04.028.

\section{Suppliers}

[1] Stryker Orthopaedics: Triathlon cruciate retaining (CR) prosthesis, Fixos cannulated compression headless screws.

[2] Palacos ${ }^{\circledR}$, Heraeus medical. 